\title{
Diacerein versus chondroitin sulfate in treatment of adult Kashin- Beck Disease: an intervention trial in Heilongjiang Province, China
}

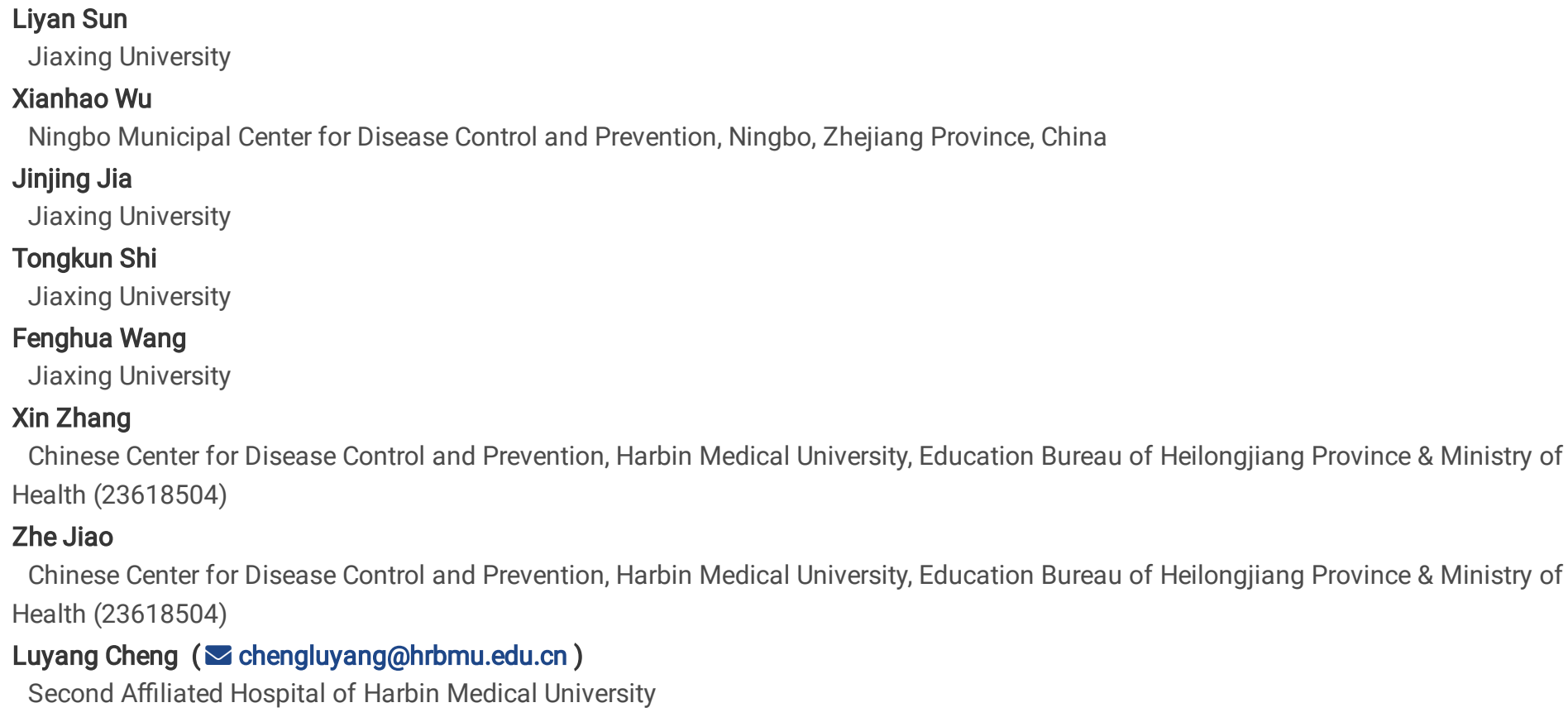

\section{Research Article}

Keywords: Kashin-Beck disease, diacerein, chondroitin sulfate, therapeutic effect, pain

Posted Date: May 12th, 2022

DOI: https://doi.org/10.21203/rs.3.rs-1244702/v2

License: (c) (i) This work is licensed under a Creative Commons Attribution 4.0 International License. Read Full License 


\section{Abstract}

Background: Kashin-Beck disease (KBD) is a kind of rare disease and currently only spread across 13 provinces from southwest to northeast China, including Tibet, Qinghai, Sichuan, Shaanxi, Heilongjiang and so on. The aim of this trial was to evaluate and compare therapeutic effects of diacerein and chondroitin sulfate on adult KBD.

Methods: Total 308 KBD patients were divided into two groups that received chondroitin sulphate (Group A) and diacerein (Group B) for 24 weeks. Data were collected at 0 (baseline), 12 (primary end point) and 24 weeks (secondary end point) to calculate the proportion of patients with effective therapeutic effect and overall improvement rate (primary efficacy parameters), WOMAC pain and stiffness scores (secondary efficacy parameters). Blood sample was collected to measure liver and renal function indexes.

Results: Two primary efficacy parameters in group $B$ at primary end point were significantly higher than those in group $A(P=0.021$, $P=0.007)$, but no statistical differences were seen in these two primary efficacy parameters between two groups at secondary end point or between primary and secondary end points in same group (all $\mathrm{P}>0.05$ ). In both groups, with the prolongation of treatment time, WOMAC pain and stiffness scores decreased significantly (all $P<0.001$ ), but no significant differences were seen between primary and secondary end points (all $\mathrm{P}>0.05$ ). In addition, in both groups, the occurrences of total adverse events were relatively low and no side effect on liver function was seen. Diacerein also had no side effect on renal function.

Conclusion: For treatment of adult KBD, both diacerein and chondroitin sulfate were effective, and diacerein might be effective and stronger than chondroitin sulfate. Taking into account both efficacy and safety, the optimal intervention time of diacerein for adult KBD patients was 12 weeks.

\section{Background}

Kashin-Beck disease (KBD) is an endemic, deformed and special type of osteoarthritis (OA) involving with growth plate and articular cartilage, and it mainly occurs in children aged 5-15 years old ${ }^{1}$. The main clinical manifestations of KBD include joint pain, enlarged and deformed joints and limb muscle atrophy. Severe cases present as short stature, even dwarfism ${ }^{2}$. KBD spread across 13 provinces from southwest to northeast China, including Tibet, Qinghai, Sichuan, Shaanxi, Heilongjiang and so on, and has crippled and stunted the growth of hundreds of people in China ${ }^{3}$.

KBD can be prevented but treated difficultly. So, Chinese government has launched a massive effort and series of etiotropic measures to prevent and control KBD ${ }^{4}$, and recent monitoring data showed that except in Tibet, no new cases occurred in other $12 \mathrm{KBD}$ endemic provinces and autonomous regions ${ }^{3}$. In 1960s and 1970s, the incidence of KBD was about $60 \%-80 \%$, and a large number of adult KBD patients aged from 40 to 70 years old were left behind from that period. KBD is a teratogenic and disabling disease, and can affect patients' ability to work and live. So, how to conduct effective treatment for adult KBD patients has become one of major public health problems in current KBD endemic areas.

Refer to $\mathrm{OA}$, currently available treatments for KBD include nonspecific interventions, pharmacologic management of symptoms and surgical treatment ${ }^{4}$. Among these treatment methods, oral drug therapy is widely used and two main categories of drugs were usually chosen: disease-modifying OA drugs (DMOADs) and non-steroidal anti-inflammatory drugs (NSAIDs). NSAIDs are heavily used to alleviate KBD patients' pain because these agents are inexpensive and easy to get, although they are well known to accompany by a high risk of adverse events, such as serious gastro-intestinal and vascular injuries ${ }^{5}$. Unlike NSAIDs, the DMOADs (such as chondroitin sulfate) have been advocated as safe and effective choice for treatment of $\mathrm{KBD}^{6}$. The results of previous studies showed that compared with placebo, chondroitin sulfate can effectively reduce knee joint space narrowing and the incidence of joint stiffness in adults $\mathrm{KBD}_{\text {patients }}{ }^{7}$ and a combination of chondroitin sulfate and glucosamine hydrochloride was more effective ${ }^{8}$.

There is strong evidence that interleukin-1 (IL-1) plays an important role in OA cartilage degradation ${ }^{9}$. As an IL-1 inhibitor, diacerein can lower the activity and reduce the content of IL-1, achieved certain treatment effects in OA. Many studies have shown that diacerein could relieve pain and slow down the progression of knee $\mathrm{OA}$ in terms of joint structure changes and symptom ${ }^{10,11}$. However, as far as we know, no study has reported the therapeutic effect of diacerein on adult KBD.

Based on current treatment status of KBD detailed above, a 24-week study of continuous medicine intervention was conducted. The primary aim of this trial was to verify and evaluate therapeutic effect of diacerein on KBD. At the same time, the therapeutic effect of diacerein and chondroitin sulfate on adult KBD patients was compared. 


\section{Design And Methods}

\section{Study design}

This study was conducted as a prospective and contrastive intervention trial with a follow-up period of 24 weeks. According to the efficacy, dose and safety of diacerein in the treatment of hand and knee OA reported in literatures ${ }^{12-14}$, although patients were followed up 24 weeks, 12 weeks was the primary end point and 24 weeks was the secondary end point. Questionnaire and physical examinations were conducted once every 12 weeks. The primary efficacy parameters were assessed at primary and secondary end points, and the secondary efficacy parameters were assessed at baseline, 12 weeks and 24 weeks.

Subjects in this trial were grouped according to their own health situation, medication history and contraindications of drug use. No placebo was included because the constraint of Administration Village Committee. Subjects were assigned to receive chondroitin sulfate or diacerein for 24 weeks.

\section{The selections of test sites}

Heilongjiang Province is a historically severe KBD endemic area and located in northwestern China. In accordance with principles of matching natural and social factors, such as geologic environments, economic situations and educational standards, towns were included if they met the following eligibility criteria: (1) the monitoring data of KBD in recent 30 years were complete; (2) historically severe KBD endemic areas; (3) the X-ray detective rate of KBD in children was < $3 \%$ in recent 3 years; (4) the number of long-term residents above 45 years old were more than 50 . Ultimately, five towns were chosen as our test sites, they were Fanrong Town, Fulu Town, Longanqiao Town, Shaowen Town of Fuyu County and Lianghe Town of Shangzhi City.

\section{Patients}

A simple questionnaire was performed and data on demographic variables (age and sex) for all residents who were living in abovementioned towns were gained firstly. Next, radiologic and clinical examinations of both hands and/or feet were performed for villagers aged 45-70 years in order to screen adult KBD patients. Adult KBD patients were diagnosed and those in degree I and II were selected in according to the Diagnosis of Kashin-Beck Disease (WS/T 207-2010 ${ }^{15}$ ).

After then, standard questionnaire and physical examinations were conducted for all selected KBD patients in degree I and II. Standard questionnaire mainly included health status, previous medication history, WOMAC pain and stiffness subscales or Assessment for Therapeutic Efficacy on Kashin-Beck Disease scale (WS/T 79-20116). Physical examinations included measurements of patients' height, weight, liver and renal function, etc. The heights and weights of patients were measured using height and weight scales. Body mass index (BMI) was calculated as the weight in kilograms divided by the square of the individual's height in meters. According to World Health Organization (WHO) criteria, the BMls were categorized into four grades: underweight (BMl<18.50 kg/m²), normal $\left(18.5 \leq \mathrm{BMl}<25 \mathrm{~kg} / \mathrm{m}^{2}\right)$, overweight $\left(25 \leq \mathrm{BMl}<30 \mathrm{~kg} / \mathrm{m}^{2}\right)$ and obese $\left(\mathrm{BMl} \geq 30.00 \mathrm{~kg} / \mathrm{m}^{2}\right)$.

KBD patients aged 45-70 years in degree I and II would be enrolled in this trial if they had lived in our test sites since they were children (6 years old). Individuals with other joint diseases, such as joint inflammation, metabolic bone diseases, neoplasia, osteoporosis, etc., or coexisting conditions such as a history of diabetes mellitus, stroke, emaciation, long-term use of drugs and cardiovascular, gastrointestinal, kidney, liver, respiratory diseases, or used drugs for OA/KBD in past three months and those who declined to participate in the study were excluded. Finally, 308 patients entered the study cohort.

\section{Group setting and interventions}

A total of 308 KBD patients were divided into two different groups (154 in group A and 154 in group B). On the basis of the literatures ${ }^{12-14}$ and drug instructions, subjects in group A and group B were assigned to receive chondroitin sulfate tablet (300mg once, three times daily) and diacerein capsule (50mg once, twice daily), respectively. Drinking was not allowed during medical treatment. Patients should stop taking drugs immediately as soon as suspicious side effects appeared.

\section{The collection of blood sample}

At 0, 12 and 24 weeks, the morning fasting blood samples of subjects (not less than $3 \mathrm{~mL} /$ person) were collected in 5 -mL tubes with or without $10 \%$ ethylene diamine tetraacetic acid, then left at room temperature for $1-2 \mathrm{~h}$. After that, the blood samples were centrifuged at $3000 \mathrm{rpm}$ for $10 \mathrm{~min}$ to separate plasma or serum. The plasma and serum were dispensed into $120 \mu \mathrm{L}$ aliquots into microcentrifuge tubes that were stored at $-80^{\circ} \mathrm{C}$ until assay. 


\section{Follow-up}

The subjects were followed up every 4 weeks by doctors from township hospital. The main jobs of each follow-up were to record amount of drug use and occurrence of AEs or SAEs. Meanwhile, drugs of next phase were distributed to subjects with good compliance. The compliance of subjects was mainly assessed by following up their medication use, including whether they taken drugs according to the instructions, the amount of drugs left over from this cycle and whether they taken other drugs at the same time.

\section{Efficacy assessment}

In this trial, Assessment for Therapeutic Efficacy on Kashin-Beck Disease (WS/T 79-2011) ${ }^{16}$ was applied to evaluate therapeutic effect of drugs, and the proportion of patients with effective therapeutic effect and overall improvement rate were calculated and set as primary efficacy parameters. Firstly, joint dysfunctions including joint rest pain (0 to 2 points), joint kinesthetic pain (0 to 2 points), morning stiffness ( 0 to 2 points), maximum walking distance ( 0 to 2 points) and lower limb mobility ( 0 to 2 points) before and after treatment were evaluated. Then, the recovery rates of joint functions after interventions were calculated using above joint dysfunctions scores. The therapeutic effects of drugs were judged to be significant, effective or invalid when the recovery rate of joint functions $\geq 70 \%, 30 \%-70 \%$ and $<30 \%$, respectively. Afterwards, the proportion of patients with effective therapeutic effect was calculated and compared. Finally, the proportion of patients with both significant and effective therapeutic effects (i.e. overall improvement rate) after interventions in different groups was gained and compared.

The WOMAC scale is a validated questionnaire addressing severity of joint pain (five questions), stiffness (two questions) and limitation of physical function (seventeen questions) of OA, researchers can use the whole system or choose parts of it. In this trial, the mean changes in WOMAC pain and stiffness subscales were chosen as the secondary efficacy parameters.

\section{Safety evaluations}

Safety evaluations in this trial included occurrences of AEs, SAEs at each visit and changes of liver and renal functions at 0,12 and 24 weeks. AEs were mainly listed in the package inserts of drugs, including diarrhea, stomach upset, nausea, dry mouth and dizziness. SAEs were defined as any fatal or life-threatening clinical experience or disabling event, or requiring long-term hospitalization, whether or not it is judged to be related to treatments.

After multiple quality control corrections, eight liver function indexes including albumin (ALB, 34-48 g/L), alkaline phosphatas (ALP, 30-90 $\mathrm{U} / \mathrm{L}$ ), alanine amino- transferase (ALT, 0-40 U/L), aspartate aminotransferase (AST, 0-40U/L), direct bilirubin (DBIL, 1-6.8 umol/L), $\gamma-$ glutamyl transpeptadase (GGT, 7-50 U/L), total bilirubin (TBIL, 5-21 umol/L), total protein (TP, 65-85 g/L) and four renal function indexes including urea (UREA, 1.8-7.5 mmol/L), creatinine (CREA, 35-79 umol/L), micro albumin (mALB, <20mg/L), N-acetyl-ß-D-glucosidase (NAG, 0.3- 14.6 $\mathrm{U} / \mathrm{L}$ ) were measured by use of automatic biochemical analyzer. Reagents for all indexes were purchased from Ningbo Meikang Biotechnology Co., Ltd., Ningbo, China.

\section{Quality control}

Our study was carried out by a well-trained research staff along with doctors, and they were trained before the trial. The training module contents included purpose of this trial, study procedures and how to implement the questionnaire, etc. The instruments used in trial were standardized by local technical supervision bureau. Additionally, to unify various instrument operation methods and formulate standard operation procedures so that the team could complete all diagnosis and follow-up tasks according to unified standard.

Three exports or technologists read same X-ray image and provide their own evaluations based on the Diagnosis of Kashin-Beck Disease (WS/T207-2010) ${ }^{15}$ to our team. The diagnosis of KBD patients was finalized by our team according to views of these experts.

A health education program was implemented to our subjects. The purpose and significance of treatment were clarified three times so that subjects could actively cooperate with treatment. Patients changed groups without permission and terminated trial unauthorized were recorded in order to control the rate of loss of follow-up strictly. The corresponding data of subjects withdrew from the trial should be handled statistically.

\section{Research ethics and patient consent}

The trial was approved by the Human and Ethics Committee for Medical Research of Center for Endemic Disease Control, Chinese Center for Disease Control and Prevention, Harbin Medical University and the Administration Village Committee in accordance with the Helsinki Declaration. The trial was registered complementally on 31/10/2020, and the registration number in the Chinese Clinical Trial Registry is 
ChiCTR2000039600 (http://www.chictr.org.cn). The purpose, significance, intended diagnostic and therapeutic methods, dosage and duration of medication and possible AEs or SAEs of this trial were informed to all subjects when they were enrollment in, after then, written informed consents were obtained from them.

\section{Statistics}

Data were analyzed by using SPSS software (version 22.0 SPSS Inc., Chicago IL). Basic characters, BMI, X-ray diagnosis of patients, primary efficacy parameters and other categorical variables were analyzed with Chi-square test. Data of WOMAC pain and stiffness scores was expressed as mean \pm standard deviation and analyzed by use of Two-way Repeated Measures Analysis of Variance. Intent-to-treat (ITT) analysis was applied in this study. All tests were two-tailed, and $P<0.05$ was considered statistically significant.

\section{Results}

\section{Basic characteristics, BMI and X-ray diagnosis of patients}

Among 308 subjects, 24 (15.58\%) in group A and 38 (24.68\%) in group B withdrew from the study at primary end point; 49 (31.82\%) in group A and 75 (48.70\%) in group B failed to complete the trial at secondary end point, and the reasons for loss of follow-up were shown in Fig. 1.

Basic characteristics of subjects including age and sex were described in Table 1. All basic characteristics were balanced and comparable between two groups at baseline (all $P>0.05$, Table 1). At the same time, there were no significant differences in BMI and X-ray diagnosis of subjects between two groups at the beginning of trial (all $P>0.05$, Table 1 ).

Table 1

Basic characteristics, BMI and X-ray diagnosis of subjects in two groups at baseline

\begin{tabular}{|c|c|c|c|c|}
\hline Characteristic & Group $A(N=105)$ & Group $B(N=79)$ & $Z$ & $P$ \\
\hline Age(years) & $57.93 \pm 4.42$ & $57.04 \pm 6.03$ & 1.855 & 0.248 \\
\hline Sex, M/F & $57 / 48$ & $42 / 37$ & 0.023 & 0.88 \\
\hline \multicolumn{5}{|l|}{$\mathrm{BMI}\left(\mathrm{kg} / \mathrm{m}^{2}\right)$} \\
\hline Underweight & $1(0.95 \%)$ & $1(1.27 \%)$ & \multirow[t]{4}{*}{1.053} & \multirow[t]{4}{*}{0.788} \\
\hline Normal & $57(54.29 \%)$ & $37(46.84 \%)$ & & \\
\hline Overweight & $37(35.24 \%)$ & $33(41.77 \%)$ & & \\
\hline Obese & $10(9.52 \%)$ & $8(10.13 \%)$ & & \\
\hline \multicolumn{5}{|l|}{ X-ray diagnosis } \\
\hline Degree I & $84(80.00 \%)$ & $62(78.48 \%)$ & \multirow[t]{2}{*}{0.063} & \multirow[t]{2}{*}{0.801} \\
\hline Degree II & $21(20.00 \%)$ & $17(21.52 \%)$ & & \\
\hline
\end{tabular}

\section{Evaluations of primary efficacy parameters}

At primary end point, the proportion of patients with effective therapeutic effect $(P=0.021)$ and overall improvement rate in group $\mathrm{B}(P=$ 0.007) were significantly higher than those in group A. However, there were no statistical differences in the proportion of patients with effective therapeutic effects $(P=0.151)$ and overall improvement rates $(P=0.301)$ between two groups at secondary end point. In both groups, no statistical differences were seen in the proportions of patients with effective therapeutic effects $\left(P_{\text {Group A }}=0.121, P_{\text {Group B }}=0.729\right)$ and overall improvement rates ( $\left.P_{\text {Group A }}=0.400, P_{\text {Group B }}=0.509\right)$ between primary and secondary end points. The data was present in Fig. 2.

\section{Comparison of Western Ontario and McMaster Universities Osteoarthritis (WOMAC) pain and stiffness scores before and after interventions}

The results of analysis of Student residuals showed that data of each group obeyed normal distribution and had no abnormal value (all $P>$ 0.05). The interaction between treatment and time had no significant effect on WOMAC pain $[\mathrm{F}(2,77)=0.924, P=0.401]$ and stiffness scores $[F(2,77)=0.617, P=0.434]$. Therefore, it was necessary to interpret main effects of treatment and time. 
There were no statistical differences in main effects of treatments of WOMAC pain $[F(1,78)=0.487, P=0.487]$ and stiffness scores $[F(1$, $78)=0.604, P=0.439$ ] between two groups. But $95 \%$ confidence intervals $(\mathrm{Cl})$ for differentials in WOMAC pain and stiffness scores between two groups (group B minus group A) were basically zero axisymmetric at baseline, and were mostly present in the left of axis of symmetry, showing left-biased distribution at primary and secondary end points. The data was shown in Table 2.

Table 2

95\% confidence intervals for differences in WOMAC pain and stiffness scores between two groups at each time point.

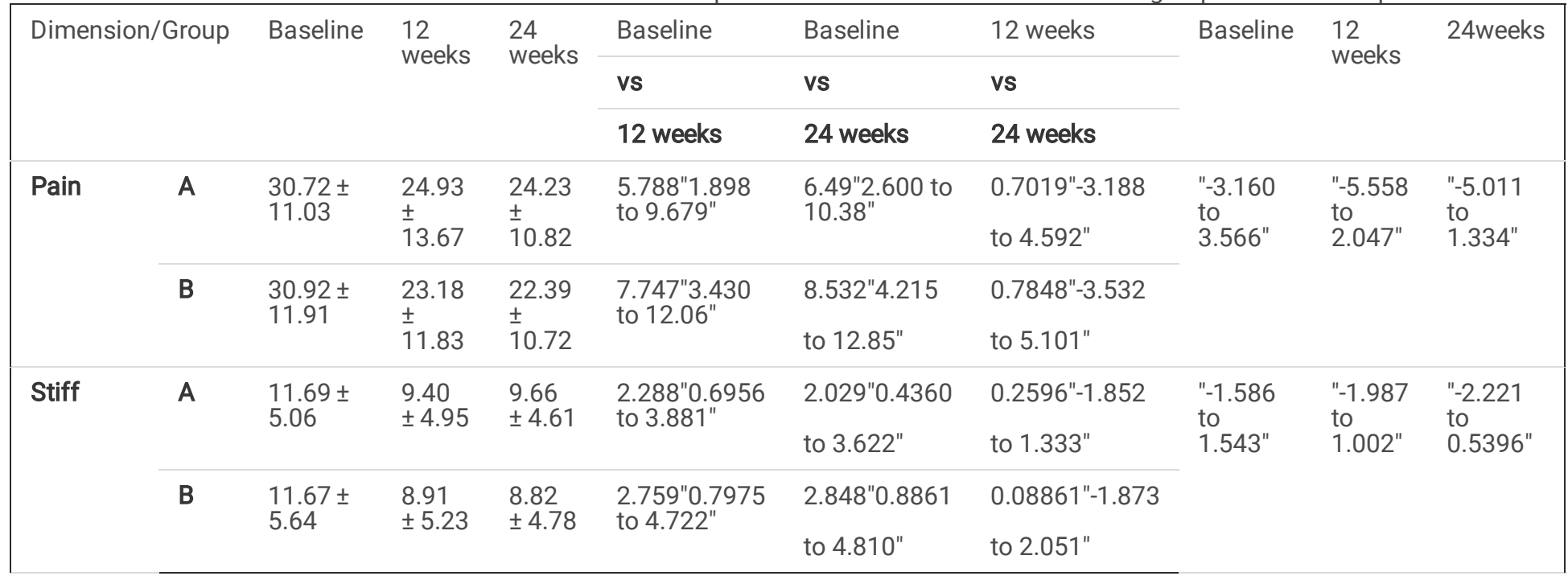

There were significant differences in main effects of times of WOMAC pain $[F(2,77)=48.553, P<0.0001]$ and stiffness scores $[F(2,77)=$ 17.713, $P<0.0001]$. In both groups, significant differences were seen in WOMAC pain ( $\left.P_{\text {Group A }}=0.0001, P_{\text {Group } B}<0.0001\right)$ and stiffness scores $\left(P_{\text {Group A }}=0.0012, P_{\text {Group B }}=0.0006\right)$. In group A, compared to baseline, WOMAC pain and stiffness scores at primary $\left(P_{\text {pain }}=0.0015\right.$, $\left.P_{\text {stiffness }}=0.0023\right)$ and secondary end points $\left(P_{\text {pain }}=0.0003, P_{\text {stiffness }}=0.0082\right)$ decreased obviously. The differentials of WOMAC pain scores were 5.79 ( $95 \% \mathrm{Cl}: 1.90$ to 9.68 , baseline VS primary end point) and 6.49 ( $95 \% \mathrm{Cl}: 2.60$ to 10.38 , baseline VS secondary end point), and the differentials of WOMAC stiffness scores were 2.29 ( $95 \% \mathrm{Cl}: 0.70$ to 3.88 , baseline VS primary end point) and 2.03 ( $95 \% \mathrm{Cl}: 0.44$ to 3.62 , baseline VS secondary end point). In group B, WOMAC pain and stiffness scores at primary $\left(P_{\text {pain }}<0.0001, P_{\text {stiffness }}=0.003\right)$ and secondary end points $\left(P_{\text {pain }}<0.0001, P_{\text {stiffness }}=0.0021\right)$ also decreased clearly. The differentials of WOMAC pain scores were $7.75(95 \% \mathrm{Cl}$ : 3.43 to 12.06 , baseline VS primary end point) and 8.53 ( $95 \% \mathrm{Cl}: 4.22$ to 12.85 , baseline VS secondary end point), and the differentials of WOMAC stiffness scores were 2.76 ( $95 \% \mathrm{Cl}: 0.80$ to 4.72 , baseline VS primary end point) and 2.85 ( $95 \% \mathrm{Cl}: 0.89$ to 4.81 , baseline VS secondary end point). There were no significant differences in WOMAC pain and stiffness scores between primary and secondary end points in both groups. The detail was shown in Table 2 and Table 3.

Table 3

Comparison of WOMAC pain and stiffness scores between two groups at baseline, first and secondary end points

\begin{tabular}{|c|c|c|c|c|c|c|c|c|c|c|c|}
\hline \multicolumn{2}{|c|}{$\begin{array}{l}\text { Dimension/ } \\
\text { Group }\end{array}$} & \multirow[t]{3}{*}{ Baseline } & \multirow[t]{3}{*}{12 weeks } & \multirow[t]{3}{*}{24 weeks } & \multirow[t]{3}{*}{$\mathrm{P}_{\text {time }}$} & Baseline & Baseline & $\begin{array}{l}12 \\
\text { weeks }\end{array}$ & \multirow[t]{3}{*}{ Baseline } & \multirow[t]{3}{*}{$\begin{array}{l}12 \\
\text { weeks }\end{array}$} & \multirow[t]{3}{*}{ 24weeks } \\
\hline & & & & & & vs & vs & vs & & & \\
\hline & & & & & & $\begin{array}{l}12 \\
\text { weeks }\end{array}$ & $\begin{array}{l}24 \\
\text { weeks }\end{array}$ & $\begin{array}{l}24 \\
\text { weeks }\end{array}$ & & & \\
\hline \multirow[t]{2}{*}{ Pain } & A & $\begin{array}{l}30.72 \pm \\
11.03\end{array}$ & $\begin{array}{l}24.93 \pm \\
13.67\end{array}$ & $\begin{array}{l}24.23 \pm \\
10.82\end{array}$ & 0.0001 & 0.0015 & 0.0003 & 0.9053 & \multirow[t]{2}{*}{0.9054} & \multirow[t]{2}{*}{0.3635} & \multirow[t]{2}{*}{0.2544} \\
\hline & B & $\begin{array}{l}30.92 \pm \\
11.91\end{array}$ & $\begin{array}{l}23.18 \pm \\
11.83\end{array}$ & $\begin{array}{l}22.39 \pm \\
10.72\end{array}$ & $\begin{array}{l}< \\
0.0001\end{array}$ & $<0.0001$ & $<0.0001$ & 0.9036 & & & \\
\hline \multirow[t]{2}{*}{ Stiff } & A & $\begin{array}{l}11.69 \pm \\
5.06\end{array}$ & $9.40 \pm 4.95$ & $9.66 \pm 4.61$ & 0.0012 & 0.0023 & 0.0082 & 0.9220 & \multirow[t]{2}{*}{0.9785} & \multirow[t]{2}{*}{0.5163} & \multirow[t]{2}{*}{0.2310} \\
\hline & B & $\begin{array}{l}11.67 \pm \\
5.64\end{array}$ & $8.91 \pm 5.23$ & $8.82 \pm 4.78$ & 0.0006 & 0.0030 & 0.0021 & 0.9938 & & & \\
\hline
\end{tabular}




\section{Safety assessment}

No severe adverse events (SAEs) were seen throughout the trial according to our follow-up records. Total adverse events (AEs) were reported in 11 patients over first 12 weeks, 4 in group $\mathrm{A}$ and 7 in group $\mathrm{B}$, and no significant difference was seen between two groups $(P=0.353)$. Total AEs were reported in 21 patients over second 12 weeks, 9 in group A and 12 in group B, and no significant difference was seen between two groups $(P=0.646)$. Among all AEs, stomach upset accounted for the highest proportion, and there was significant difference in stomach upset between two groups at secondary end point $(P=0.022)$. No significant differences were seen in total AEs in both groups between primary and secondary end points $\left(P_{\text {group } A}=0.152, P_{\text {group } B}=0.336\right)$. The data was shown in Table 4 .

Table 4

The occurrences of AEs in different groups at primary and secondary end points

\begin{tabular}{|lllllllll|}
\hline SAEs & Group A & \multicolumn{3}{c}{ Group B } & \multicolumn{3}{c|}{ 12/24 weeks } & Group A/ B \\
\cline { 2 - 9 } & 12 weeks & 24 weeks & 12 weeks & 24 weeks & P(Group A) & P(Group B) & P(12 weeks) & P(24 weeks) \\
\hline Diarrhea & $0(0.00 \%)$ & $0(0.00 \%)$ & $2(2.53 \%)$ & $1(1.27 \%)$ & NA & 0.560 & 0.183 & 0.248 \\
\hline Stomach upset & $3(2.86 \%)$ & $3(2.86 \%)$ & $5(6.33 \%)$ & $9(11.39 \%)$ & 1.000 & 0.263 & 0.253 & 0.022 \\
\hline Vomiting & $0(0.00 \%)$ & $3(2.86 \%)$ & $0(0.00 \%)$ & $1(1.27 \%)$ & 0.081 & 0.500 & NA & 0.424 \\
\hline Dry mouth & $1(0.95 \%)$ & $2(1.90 \%)$ & $0(0.00 \%)$ & $0(0.00 \%)$ & 0.561 & NA & 0.384 & 0.217 \\
\hline Dizziness & $0(0.00 \%)$ & $1(0.95 \%)$ & $0(0.00 \%)$ & $0(0.00 \%)$ & 0.316 & NA & NA & 0.384 \\
\hline Total & $4(3.81 \%)$ & $9(11.39 \%)$ & $7(6.67 \%)$ & $12(15.19 \%)$ & 0.152 & 0.336 & 0.353 & 0.646 \\
\hline
\end{tabular}

At primary end point, the proportion of patients with abnormal DBIL in group A was significantly higher than that in group B $(P=0.012)$. Besides DBIL at primary end point, no significant differences were seen in other liver function indexes between two groups at other time points. At same time, there were no significant differences in all 8 liver function indexes in both groups before and after interventions (Fig. 3).

At baseline, the proportion of patient with abnormal mALB in group A (38.2\%) was significantly higher than that in group B $(19.0 \%, P=$ 0.004). At secondary end point, the proportion of patients with abnormal UREA in group $A(3.8 \%)$ was significantly lower than that in group $\mathrm{B}(11.4 \%, P=0.047)$. Besides above two indexes, no significant differences were seen in the proportions of abnormal patients with other renal function indexes between two groups at other time points (all $P>0.05$, Fig. 4).

In group $A$ : the proportions of patients with abnormal UREA and CREA at secondary end point were significantly lower than those at primary time point $\left(P_{\text {UREA }}=0.014, P_{\text {CREA }}=0.003\right)$ and baseline $\left(P_{\text {UREA }}=0.008, P_{\text {CREA }}=0.005\right)$; the proportions of patients with abnormal $m A L B$ at primary $(P=0.002)$ and secondary end points $(P<0.001)$ were significantly lower than that at baseline; the proportion of patient with abnormal NAG at secondary end point was significantly higher than those at primary end point $(P=0.031)$ and baseline $(P=0.031)$. Differently, in group B, no significant differences were seen in the proportions of abnormal patients with four renal function indexes throughout the trial (all $P>0.05$, Fig. 4).

\section{Discussion}

From 2006 to 2017, a treatment program for adult KBD patients in historical severe KBD endemic areas including Sichuan, Tibet, Qinghai, Inner Mongolia, Jilin, Heilongjiang, Gansu, Shaanxi, Shanxi, Shandong and Hebei provinces (autonomous regions) was carried out under the support of Chinese government. NSAIDs, DMOADs, vitamins, proprietary Chinese medicines were adopted alone or jointly according to actual situation of each endemic area. Unfortunately, the therapeutic effect of drugs used in this program was difficult to accurately evaluate due to different drug selection and treatment cycle. Meanwhile, the results showed that oral drugs were better for adult KBD patients in degree I and II and not ideal for adult KBD patients in degree III. Based on this program, adult KBD patients in degree I and II were selected as our subjects in this study.

Assessment for Therapeutic Efficacy on Kashin-Beck Disease (WS/T 79-2011) ${ }^{16}$ stipulates principle and method of determining clinical treatment effect, which can assess joints status by evaluating daily living and working functions of KBD patients. The standard was specific and reliable, and has been used widely to evaluate the treatment of KBD ${ }^{17-19}$. Previous results of our team in 2010 indicated that chondroitin sulfate was effective in treating adult $\mathrm{KBD}^{7}$, and the result was verified once again in this trial. During 24 -week interventions, nearly $20 \%$ of patients in group A showed effective and/or significant therapeutic effects. Encouragingly, overall improvement rates of 
patients in group B were consistently higher than $30 \%$ at primary and secondary end points. In first 12-week, the therapeutic effects (both the proportion of patients with effective therapeutic effect and overall improvement rate) of diacerein were much better than chondroitin sulfate. In second 12-week, the therapeutic advantage of diacerein remained, but became less pronounced. And from 12 to 24 weeks, there was little increase in the proportions of patients with effective therapeutic effects and overall improvement rates in two groups, namely the differences in therapeutic effects of two drugs between two end points were not obviously. On the one hand, above results suggested that therapeutic effect of diacarin for KBD was stronger than that of chondroitin sulfate. On the other hand, therapeutic effects of both two drugs could be achieved better after 12-week interventions, and could be maintained but not enhanced significantly with the extension of treatment time.

The WOMAC scale is used widely to assess the severity of OA or KBD and the efficacy of treatment based on associated symptoms and signs $^{8,12}$. In scale, the structure and function of hip and knee were evaluated by pain, stiffness and joint function, and the functional description was mainly for lower limbs ${ }^{13,20}$. However, in KBD patients, the interphalangeal, wrist and ankle were most frequently affected among all involved joints because the disease occurs in childhood and mainly harmed epiphysis and metaphysis of phalanges ${ }^{21}$. So, depending on patients' main symptoms and involved joints, WOMAC pain and stiffness subscales were used as secondary efficacy parameters in this trial.

There were no statistical differences in WOMAC pain and stiffness scores between two groups at all three time points. But from baseline to primary and secondary end points, 95\% Cl for differentials in WOMAC pain and stiffness scores between two groups went from basically zero axisymmetric to left-biased distribution, indicating WOMAC pain and stiffness scores of two groups changed from essentially equal to that of group B being less than group A. Therefore, above results implied that therapeutic effects of diacarin for pain and stiffness in KBD were slightly better than chondroitin sulfate. In both groups, with the prolongation of treatment time, WOMAC pain and stiffness scores decreased significantly, but there were no significant differences in WOMAC pain and stiffness scores between primary and secondary end points. The change trends of WOMAC pain and stiffness scores were consistent with therapeutic effects of drugs in above section. These two results corroborated each other and proved together that 12 weeks was a suitable intervention time for diacerein and chondroitin sulfate. Besides, in $95 \% \mathrm{Cl}$ terms, both drugs improved pain better.

The occurrences of AEs, SAEs and changes of liver and renal functions at each visit were monitored to evaluate safety of drugs used in this study. Firstly, no SAEs were found in this study. Secondly, the occurrences of total AEs in both groups were relatively low, and no significant differences were seen between two groups at same time point or between two end points at same group. But after 24-week treatment, the incidence of stomach upset caused by diacerein was clearly higher than that caused by chondroitin sulfate. Thirdly, diacerein had no side effects on liver and renal functions at any end point. Finally, chondroitin sulfate had no side effects on liver function and could the proportion of patients with abnormal UREA, CREA and mALB decrease apparently, but the proportion of patients with abnormal NAG increased. NAG is a sensitive indicator of kidney injury, the level of NAG can be more than 10 times higher than the upper limit of normal value when renal function is impaired. Among 7 patients with abnormal NAG, 2 patients had high NAG levels and which were slightly higher than the upper limit of normal value; remaining 5 patients had lower NAG levels and which were lower than the lower limit of normal value (data was not provided in this article). In other words, none of above changes of NAG had clinical significance. In total, all above results showed that the safety of drugs used in this study were better, especially at primary end point.

The rate of patients who lost to follow-up at secondary end point was relatively high and this would reduce the reliability of results. In order to analyze possible impact of withdraw on results, a sensitivity analysis was conducted. According to information obtained in the field and results of sensitivity analysis, possible reasons for loss of follow-up including following three points: firstly, at baseline of the trial, it was busy farming season and most villagers were farming at home, so subjects were easy to recruit. However, at secondary end point of the trial, it was slack farming season, and some relatively younger subjects went to work in other places, so they could not return to their own villages for follow-up. This was consistent with result of sensitivity analysis that the age of subjects who lost to follow-up in group $B$ (56.55 $\pm 7.13)$ was younger than that in group $\mathrm{A}(59.53 \pm 5.60, P=0.0148$, S-Table 1$)$. Secondly, some KBD patients did not know two drugs used in this trial and feel its efficacies, especially lessening pain were not obvious, so they give up taking the drug and withdrew from the trial, and data supporting this hypothesis were shown in S-Table 2. In both groups, the WOMAC pain scores of patients who were lost to follow-up were significantly lower than those of patients who completed the trial $\left(P_{\text {Group A }}=0.0465, P_{\text {Group B }}=0.0058\right)$. Finally, the last follow-up was completed in mid-December 2019, the COVID-19 outbreak prevented our team from making any further investigation of lost subjects and evaluating the subsequent effects of DC after 6-week and 12-week treatment-free observation period.

Besides the rate of subjects who lost to follow-up was relatively high, this trial still has other limitations. First, no placebo group was set due to the restrictions of Administration Village Committee, which would reduce the accuracy of the study. Second, blind grouping was not applied because subjects' health status. The statistical efficiency would improve if blind grouping is used. Last, all subjects were from 
Heilongjiang Province and there might be regional limitations. Therefore, we will continue to conduct further study in future to verify the authenticity of this study.

\section{Conclusions}

In summary, the results showed that both diacerein and chondroitin sulfate had certain therapeutic effect on adult KBD patients and could improve pain and stiffness. 12-week interventions were suitable, drugs could work effectively and avoid side effects minimally. Compared with chondroitin sulfate, the therapeutic effect of diacerein on adult KBD might work stronger. The results of this trial suggested that diacerein could be used to treat adult KBD in KBD endemic areas in future.

\section{Abbreviations}

AEs: adverse events; ALB: albumin; ALP: alkaline phosphatas; ALT: alanine amino-transferase; AST: aspartate aminotransferase; BMI: body mass index; CREA: creatinine; DBIL: direct bilirubin; DMOADs: disease-modifying OA drugs; GGT: Y-glutamyl transpeptadase; IL-1: interleukin-1; ITT: intent-to-treat; KBD: Kashin- Beck disease; mALB: micro albumin; NAG: N-acetyl- $\beta$-D-glucosidase; NSAIDs: non-steroidal anti-inflammatory drugs; OA: osteoarthritis; SAEs: severe adverse events; TBIL: total bilirubin; TP: total protein; UREA: urea; WOMAC: Western Ontario and McMaster Universities Osteoarthritis;

\section{Declarations}

Ethics approval and consent to participate

The trial was approved by the Human and Ethics Committee for Medical Research of Center for Endemic Disease Control, Chinese Center for Disease Control and Prevention, Harbin Medical University and the Administration Village Committee in accordance with the Helsinki Declaration. The trial was registered complementally on 31/10/2020, and the registration number in the Chinese Clinical Trial Registry is ChiCTR2000039600 (http://www.chictr.org.cn).

\section{Consent for publication}

Not applicable.

\section{Availability of data and materials}

The datasets used and/or analyzed during the current study are available from the corresponding author on reasonable request.

\section{Competing interests}

All authors declare that they have no competing interests.

\section{Funding}

This study was supported by grants from Heilongjiang Provincial Postdoctoral Science Foundation (LBH-Q18073), the Major Project of Applied Technology and Development Program of Heilongjiang Province (GA18C007) and the Research Initiation Project of Jiaxing University (CD70520043).

\section{Authors' contributors}

Liyan Sun designed the trial. Liyan Sun, Xianhao Wu, Xin Zhang and Zhe Jiao participated in the investigation and collected samples. Xianhao Wu and Xin Zhang performed serum and urine assay. Tongkun Shi and Jinjing Jia analyzed the data. Fenghua Wang and Luyang Cheng searched literature. Liyan Sun and Luyang Cheng wrote and revised the manuscript. All authors read and approved the final manuscript.

\section{Acknowledgments}

We thank the Centers for Disease Control and Prevention of Shangzhi City and Fuyu County for their assistance in quality control and patient follow-up. We also gratefully acknowledge the parents and guardians of the subjects for their cooperation.

\section{References}


1. Cao J, Li S, Shi Z, Yue Y, Sun J, Chen J, Fu Q, Hughes CE, Caterson B. Articular cartilage metabolism in patients with Kaschin-Beck disease: an endemic osteoarthropathy in China. Osteoarthritis Cartilage. 2008;16(6):680-8. doi:10. 1016/j.joca.2007.09.002.

2. Stone R. Diseases. A medical mystery in middle China. Science. 2009;324(5933): 1378-81. doi: 10.1126/science.324_1378.

3. Sun LY, Cui SL, Deng Q, Liu H, Cao YH, Wang SP, Yu J. Selenium Content and/or T-2 Toxin Contamination of Cereals, Soil, and Children's Hair in Some Areas of Heilongjiang and Gansu Provinces, China. Biol Trace Elem Res. 2019; 191(2):294-9. doi:10.1007/s12011-0181620-7.

4. Sun LY, Meng FG, Li Q, Zhao ZJ, He CZ, Wang SP, Sa RL, Man WW, Wang LH. Effects of the consumption of rice from non-KBD areas and selenium supplementation on the prevention and treatment of paediatric Kaschin-Beck disease: an epidemiological intervention trial in the Qinghai Province. Osteoarthritis Cartilage. 2014;22(12):2033-40. doi:10.1016/j.joca.2014. 09.013.

5. Schjerning AM, McGettigan P, Gislason G. Cardiovascular effects and safety of (non-aspirin) NSAIDs. Nat Rev Cardiol. 2020;17(9):57484. doi:10.1038/s41569-020-0366-z.

6. Uebelhart D, Malaise M, Marcolongo R, Vathaire F, Piperno M, Mailleux E, Fioravanti A, Matoso L, Vignon E. Intermittent treatment of knee osteoarthritis with oral chondroitin sulfate: a one-year, randomized, double-blind, multicenter study versus placebo. Osteoarthritis Cartilage. 2004;12(4):269-76. doi:10.1016/ j.joca.2004. 01.004.

7. Zhang YX, Dong W, Liu H, Cicuttini F, Courten M, Yang JB. Effects of chondroitin sulfate and glucosamine in adult patients with Kaschin-Beck disease. Clin Rheumatol. 2010;29(4):357-62. doi:10. 1007/s10067-009-1311-z.

8. Yue J, Yang M, Yi S, Dong B, Li W, Yang Z, Lu J, Zhang R, Yong J. Chondroitin sulfate and/or glucosamine hydrochloride for KashinBeck disease: a cluster-randomized, placebo- controlled study. Osteoarthritis Cartilage. 2012;20(7): 622-9. doi: 10.1016/j.joca.2012.03.013.

9. Burger D, Dayer JM, Palmer G, Gabay C. Is IL-1 a good therapeutic target in the treatment of arthritis? Best Pract Res Clin Rheumatol. 2006;20(5):879-96. doi: 10.1016/j.berh.2006.06. 004.

10. Pavelka K, Bruyère O, Cooper C, Kanis JA, Leeb BF, Maheu E, Martel-Pelletier J, Monfort J, Pelletier JP, Rizzoli R, Reginster JY. Diacerein: Benefits, Risks and Place in the Management of Osteoarthritis. An Opinion-Based Report from the ESCEO. Drugs Aging. 2016;33(2):7585. doi:10.1007/s40266-016-0347-4.

11. Louthrenoo W, Nilganuwong S, Nanagara R, Siripaitoon B, Basset SC. Diacerein for the treatment of rheumatoid arthritis in patients with inadequate response to methotrexate: a pilot randomized, double-blind, placebo-controlled add-on trial. Clin Rheumatol. 2019;38(9):2461-71. doi:10.1007/s10067-019-04587-1.

12. Pavelka K, Trc T, Karpas K, Vítek P, Sedlácková M, Vlasáková V, Böhmová J, Rovenský J. The efficacy and safety of diacerein in the treatment of painful osteoarthritis of the knee: a randomized, multicenter, double-blind, placebo- controlled study with primary end points at two months after the end of a three- month treatment period. Arthritis Rheum. 2007;56(12): 4055-64. doi: 10.1002/ art.23056.

13. Louthrenoo W, Nilganuwong S, Aksaranugraha S, Asavatanabodee P, Saengnipanthkul S, Thai Study Group. The efficacy, safety and carry-over effect of diacerein in the treatment of painful knee osteoarthritis: a randomised, double-blind, NSAID-controlled study. Osteoarthritis Cartilage. 2007;15(6):605-14. doi: 10.1016/j.joca.2007.02.021.

14. Shin K, Kim JW, Moon KW, Yang JA, Lee EY, Song YW, Lee EB. The Efficacy of Diacerein in Hand Osteoarthritis: A Double-Blind, Randomized, Placebo-Controlled Study. Clin Ther. 2013;35(4):431-9. doi: 10.1016/j.clinthera. 2013.02.009.

15. Ministry of Health of the People's Republic of China Document: Guidelines for health. Diagnosis of Kaschin-Beck Disease (WS/T 2072010), June 2, 2010.

16. Ministry of Health of the People's Republic of China Document: Guidelines for health. Assessment for therapeutic efficacy on KashinBeck Disease (WS/T 79-2011), November 22, 2011.

17. Zhang W, Bai XQ, Liu XY. Effect of drug therapy of patients with Kashin-Beck disease at Yuyang district of Yulin city from 2014 to 2018 (In Chinese). Bulletin of Disease Control \& Prevention (China). 2020; 35: 48-51.

18. Yu FF, Ren FL, Fang H, Xia CT, Guo X. The improvement of quality life of intro-articular hyaluronic acid on adults with Kashin-Beck disease (In Chinese). Chinese Journal of Endemiology (China). 2015; 34: 376-8.

19. Xing ZF, Zhang CN, Zhang Z, Shi G. Effect of chondroitin sulfate, meloxicam and vitamin family on adult Kashin-Beck disease (In Chinese). Chinese Journal of Public Health Management (China). 2014; 30: 412-3.

20. Pelletier JP, Raynauld JP, Dorais M, Bessette L, Dokoupilova E, Morin F, Pavelka K, Paiement P, Martel-Pelletier J; DISSCO Trial Investigator Group. An international, multicentre, double-blind, randomized study (DISSCO): effect of diacerein vs celecoxib on symptoms in knee osteoarthritis. Rheumatology (Oxford). 2020;59(12):3858-68. doi: 10.1093/rheumatology /keaa072. 
21. Huang Q, Zhou ZK, Ma J, Li Y, Yang X, Shen B, Yang J, Kang PD, Pei FX. The arthropathic and functional impairment features of adult Kashin-Beck disease patients in Aba Tibetan area in China. Osteoarthritis Cartilage. 2015;23(4):601-6. doi:

\section{Figures}

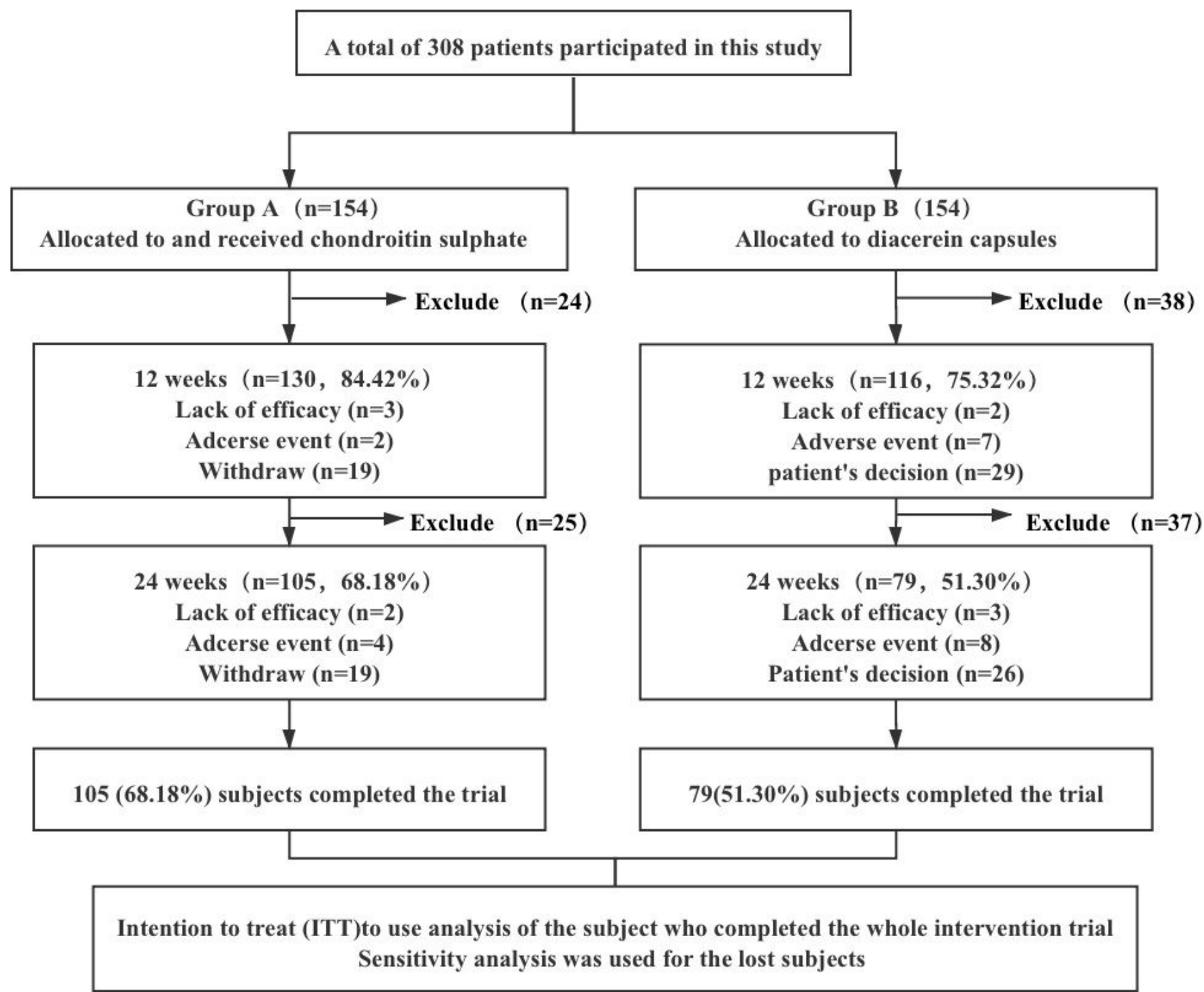

Figure 1

Flow chart of the trial. 


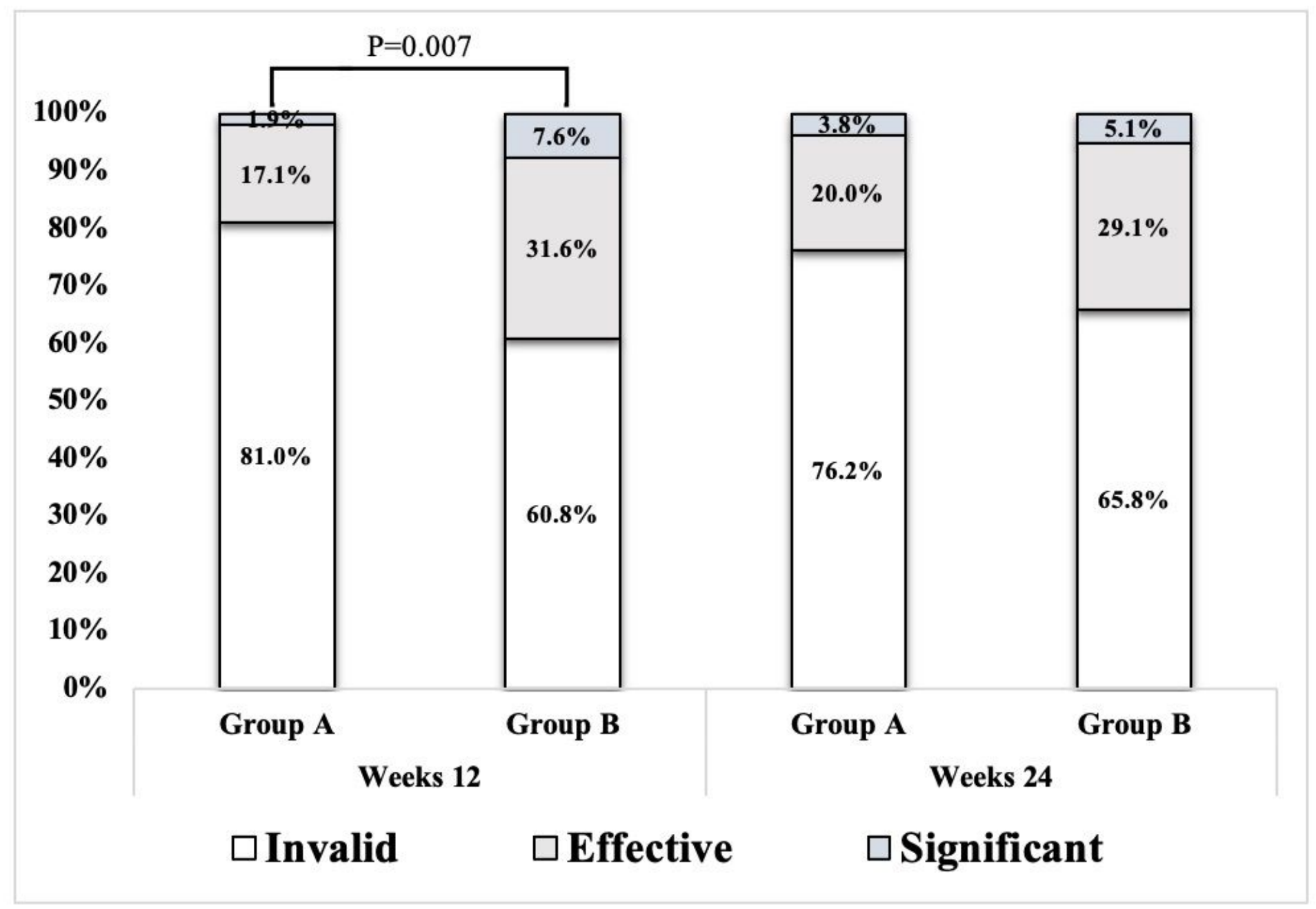

\section{Figure 2}

The proportions of patients with invalid, effective and significant therapeutic effects in each group after 12 and 24 weeks' interventions. At 12 weeks, the proportion of patients with effective therapeutic effect $(P=0.021)$ and overall improvement rate in group $\mathrm{B}(P=0.007)$ were significantly higher than those in group $\mathrm{A}$. 


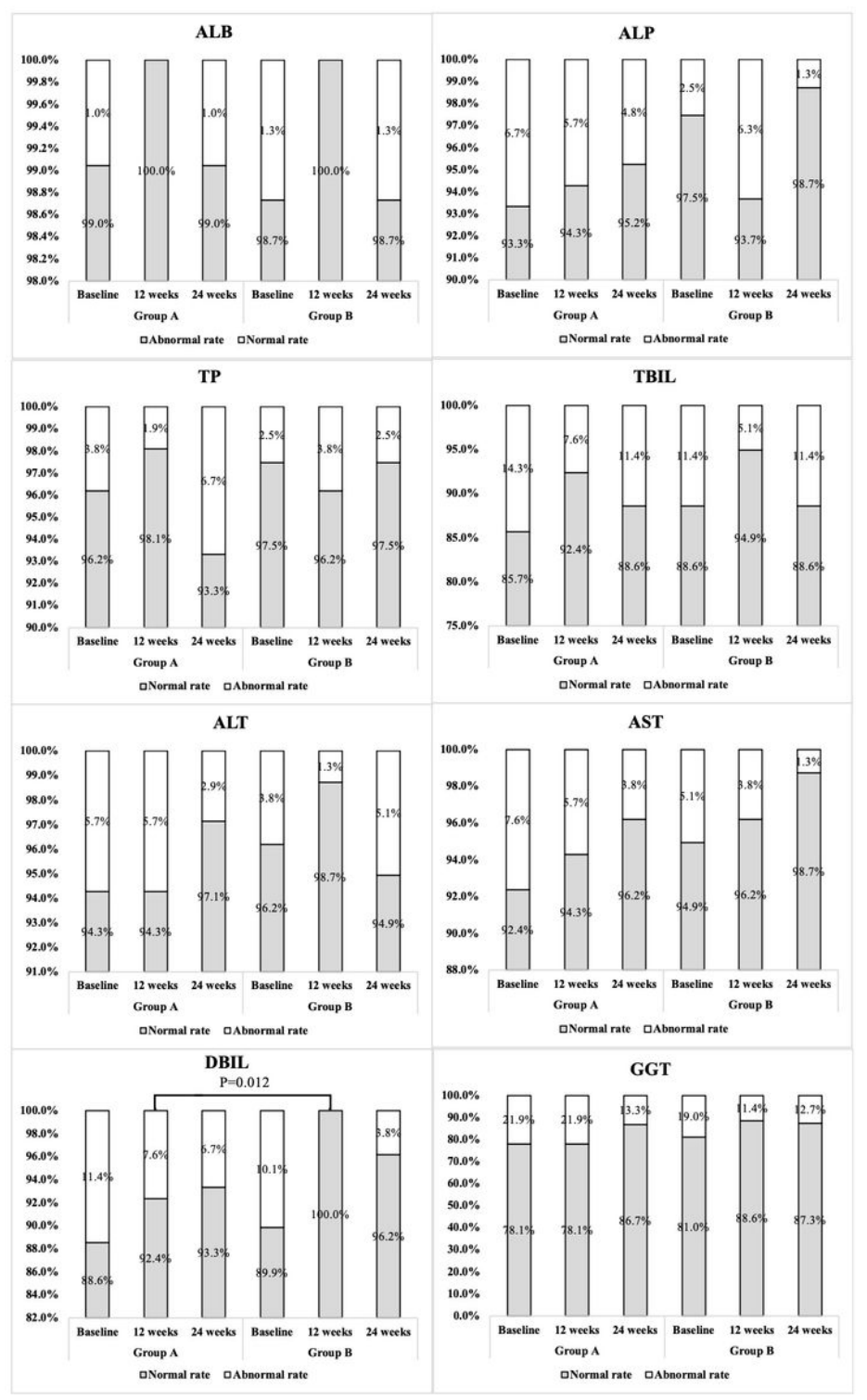

Figure 3

The proportions of patients with abnormal liver function indexes in each group at different time points. At 12 weeks, the proportion of patients with abnormal DBIL in group $A$ was significantly higher than that in group $B(P=0.012)$. 


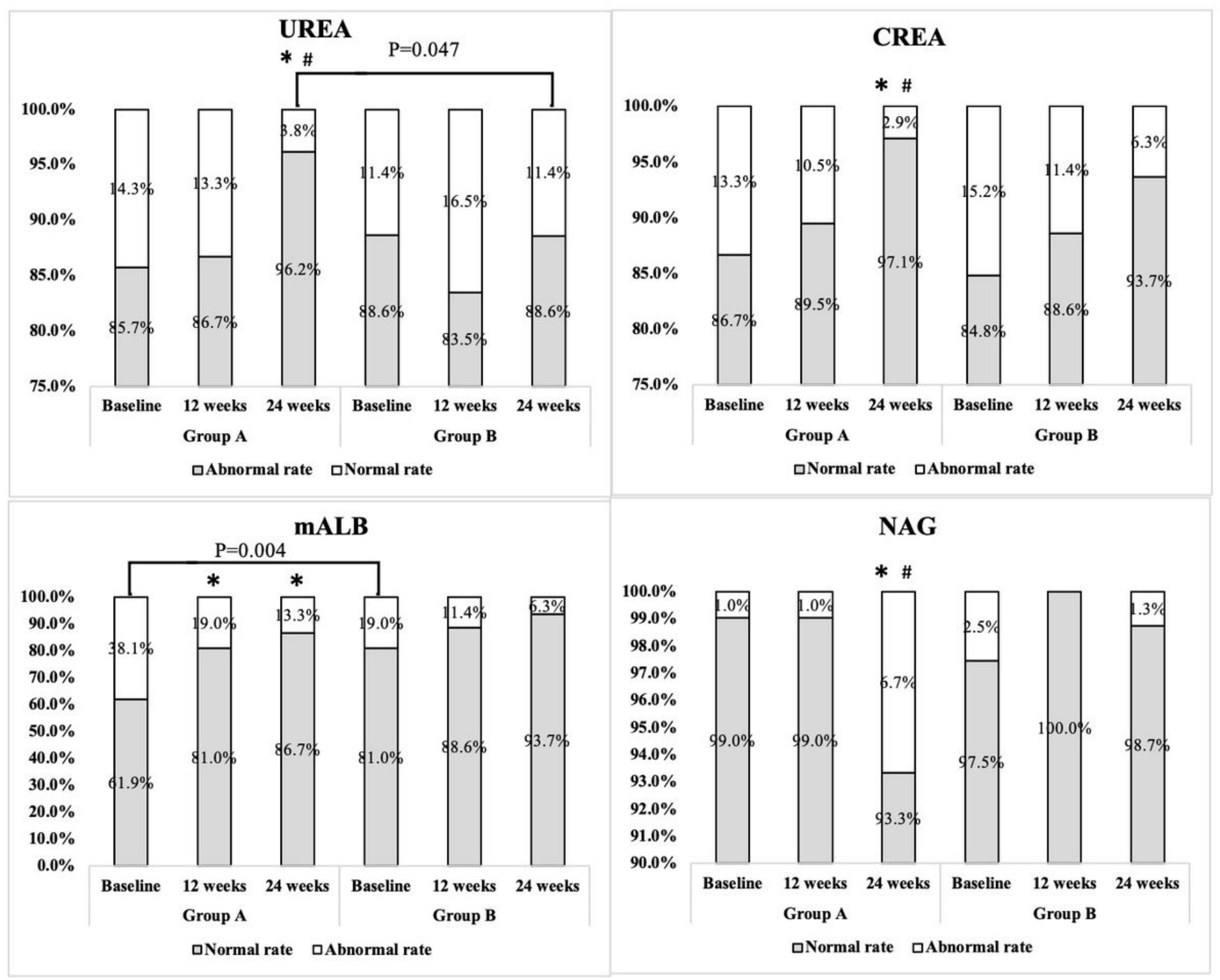

Figure 4

The proportions of patients with abnormal renal function indexes in each group at different time points. At baseline, the proportion of patient with abnormal mALB in group A was significantly higher than that in group $B(P=0.004)$. At 24 weeks, the proportion of patients with abnormal UREA in group A was significantly lower than that in group $B(P=0.047)$. In group $A$ : the proportions of patients with abnormal UREA and CREA at 24 weeks were significantly lower than those at 12 weeks $\left(P_{\text {UREA }}=0.014, P_{\text {CREA }}=0.003\right)$ and baseline $\left(P_{\text {UREA }}=0.008, P\right.$ CREA $=0.005)$; the proportions of patients with abnormal mALB at $12(P=0.002)$ and 24 weeks $(P<0.001)$ were significantly lower than that at baseline; the proportion of patient with abnormal NAG at 24 weeks was significantly higher than those at 12 weeks $(P=0.031)$ and baseline $(P=0.031) .{ }^{*}$ means $P<0.05$ compared to baseline ${ }^{\#}$ indicates $P<0.05$ compared to 12 weeks.

\section{Supplementary Files}

This is a list of supplementary files associated with this preprint. Click to download.

- STable1and2.doc 